\title{
Evaluation of Cytokine Levels and Pulmonary Function in Patients Undergoing Coronary Artery Bypass Graft
}

\section{Dear Editor,}

First, we would like to congratulate the authors by the article entitled Evaluation of cytokine levels and pulmonary function in patients undergoing coronary artery bypass graft, recently published in the Brazilian Journal of Anesthesiology ${ }^{1}$.

The importance of knowing the inflammatory status in postoperative period of patients undergoing cardiac surgery is related to the fact that, in addition to the variables shown in the study, it can influence the time of mechanical ventilation and permanence in the ICU ${ }^{1}$. As demonstrated by Kilger et al. ${ }^{2}$, patients who received hydrocortisone doses in the postoperative period of cardiac surgery had low levels of IL-6 and stayed less time in the ICU when compared with those who did not receive the treatment.

However, in postoperative period, the evaluation of brain natriuretic peptide (BNP) renders the best predictor of clinical outcomes in patients undergoing cardiac surgery when compared with inflammatory markers. This became evident in a study by Ganem et al. ${ }^{3}$, which evaluated BNP levels, IL-6, IL-8, P-selectin, ICAM and C-reactive protein, and correlated them with the immediate clinical outcomes in patients undergoing cardiac surgery. The study showed that high BNP levels are correlated with longer staying in the ICU, longer mechanical ventilation time and dobutamine use. The BNP plasmatic levels $>190 \mathrm{pg} \cdot \mathrm{mL}^{-1}$ present as an independent predictor of permanence over five days in the ICU, which is not evidenced in this period by using inflammatory factors. Thus, it is interesting to include the BNP evaluation during postoperative period in future studies in order to correlate it with extracorporeal circulation time.

As Machado et al. ${ }^{1}$, they observed that an increase in absolute values of IL- 6 are related to extracorporeal circulation time, and that variations in IL-8 levels are related to a longer surgery time. Liu et al. ${ }^{4}$ correlated such inflammatory markers with time of mechanical ventilation in children undergoing cardiac surgery and who had renal failure. The authors evidenced IL-6 and IL-8 levels significantly higher in patients who needed mechanical ventilation for a longer period.

Another important and influential factor related to inflammatory factors in those patients will be the adopted ventilation regime. Oliveira et al. ${ }^{5}$ compared cytokines levels in the bronchoalveolar lavage in two groups of mechanically ventilated patients without pulmonary disease who were inpatients in the postoperative ICU. One group used ventilation with low current volume (5-7 mL. $\left.\mathrm{kg}^{-1}\right)$ and the other one had ventilation with high current volume $\left(10-12 \mathrm{~mL} . \mathrm{kg}^{-1}\right)$. The study showed that using high current volume increases significantly IL-8 and TNF- $\alpha$ levels.

Consequently, we can conclude that even though those inflammatory markers are high primarily due to extracorporeal circulation time, once the current volume used ranged from 6 to $8 \mathrm{~mL} . \mathrm{kg}^{-1}$ in patients undergoing cardiac surgery, other factors like the time of mechanical ventilation during surgery and the ventilation strategy adopted may also contribute to alter absolute levels of those cytokines as well as clinical outcomes immediately after the postoperative period.

Soraia Genebra Ibrahim Intensive Care Center, Moinhos de Vento Hospital

Luiz Alberto Forgiarini Junior Centro Universitário Metodista (IPA);

Airways and Lungs Laboratory Hospital de Clínicas de Porto Alegre (HCPA)

Elaine Aparecida Félix Department of Anesthesy,. Universidade Federal do Rio Grande do Sul (UFRGS); Head of Anesthesiology and Perioperative Medicine Service, Hospital de Cínicas de Porto Alegre (HCPA) 


\section{REFERÊNCIAS / REFERENCES}

01. Machado LB, Negri EM, Bonafé WW, Santos LM, Malbouisson LMS, Carmona MJC - Evaluation of cytokine levels and pulmonary function in patients undergoing coronary artery bypass graft. Rev Bras Anestesiol, 2011;61(3):275-285.

02. Kilger E, Heyn J, Beiras-Fernandez A, Luchting B, Weis F - Stress doses of hydrocortisone reduces systemic inflammatory responses in patients undergoing cardiac surgery without cardiopulmonary bypass. Minerva Anestesiol, 2011;77(3):268-274.

03. Ganem F, Serrano CV, Fernandes JL et al. - Preoperative B-type natriuretic peptide, and not the inflamation status, predicts na andverse outcome for patients undergoing heart surgery. Interact Cardiovasc Thorac Surg, 2011;12(5):778-783.

04. Liu KD, Altmann C, Smits G et al. - Serum Interleukin-6 and interleukin-8 are early biomarkers of acute kidney injury and predict prolonged mechanical ventilation in children undergoing cardiac surgery: a case-control study. Crit Care, 2009;13(4):R104.

05. Oliveira RP, Hetzel MP, Silva MA, Dallegrave D, Friedman G-Mechanical ventilation with high tidal volume induces inflammation in patients without lung disease. Crit Care, 2010;14(2):R39. 